ERRATA

\title{
Expression of activated M-Ras in a murine mammary epithelial cell line induces epithelial-mesenchymal transition and tumorigenesis
}

Katherine R Ward, Kai-Xiu Zhang, Aruna M Somasiri, Calvin D Roskelley and John W Schrader

Oncogene (2004) 23, 8858. doi:10.1038/sj.onc.1207865

Correction to: Oncogene (2004) 23, 1187-1196.

doi: $10.1038 /$ sj.onc. 1207226
Owing to a typesetting error, the second author name was spelled incorrectly. The correct spelling is Kai-Xin Zhang.

\section{To be, or not to be: NF- $\kappa$ B is the answer - role of Rel/NF- $\kappa$ B in the regulation of apoptosis}

Jérôme Kucharczak, Matthew J Simmons, Yongjun Fan and Céline Gélinas

Oncogene (2004) 23, 8858. doi:10.1038/sj.onc.1207744

Corrections to: Oncogene (2003) 22, 8961-8982.

doi:10.1038/sj.onc. 1207230

Owing to a production error, the beginning of the first sentence on p. 8962 was omitted. The correct sentence should read: 'Several reports revealed that casein kinase II can also phosphorylate $\mathrm{I} \kappa \mathrm{B} \alpha$ to promote its degradation (Schwarz et al., 1996; Miyamoto et al., 1998; Romieu-Mourez et al., 2001; Shen et al., 2001; Kato et al., 2003).' The phenotype of relA-deficient mice was also listed incorrectly as $\operatorname{Rel}^{+}$at three places in the last paragraph of p. 8965. The correct description is $\operatorname{rel} A^{-1-}$. 